\title{
On Critical Orientations in the Kedem-Sharir Motion Planning Algorithm*
}

\author{
K. Kedem, ${ }^{1}$ M. Sharir, ${ }^{2.3}$ and S. Toledo ${ }^{2}$ \\ 1 Department of Mathematics and Computer Science, \\ Ben-Gurion University of the Negev, \\ Beer-Sheva 84105 , Israel \\ klara@cs.bgu.ac.il \\ 2 Department of Computer Science, Tel-Aviv University, \\ Tel-Aviv 69978, Israel \\ sharir@math.tau.ac.il \\ toledo@parc.xerox.com \\ ${ }^{3}$ Courant Institute of Mathematical Sciences, New York University, \\ New York, NY 10012, USA
}

\begin{abstract}
We discuss a technical problem arising in the motion planning algorithm of Kedem and Sharir [KS], and propose a way to overcome it without increasing the asymptotic complexity of the algorithm.
\end{abstract}

\section{Introduction}

The paper [KS], by Kedem and Sharir, studies the problem of planning a collisionfree motion (including translation and rotation) for a convex polygonal body $B$, with $k$ corners, amidst polygonal obstacles having $n$ corners altogether. More specifically, the problem is stated as follows: given initial and final placements of $B$, determine whether there is an obstacle-avoiding motion from the initial placement to the final placement and, if so, plan such a motion.

${ }^{*}$ The first author was supported by the Eshkol Grant 04601-90 from the Israeli Ministry of Science and Technology. The second author was partly supported by the Fund for Basic Research administered by the Israeli Academy of Sciences, by National Science Foundation Grants CCR-91-22103 and CCR-93-11127, and by grants from the U.S.-Israeli Binational Science Foundation, and the G.I.F., the German-Israeli Foundation for Scientific Research and Development. The third author was partly supported by the Interdisciplinary Program at Tel-Aviv University. The third author's current address is: Department of Computer Science, MIT, Boston. MA, USA. 
In what follows we assume some familiarity of the reader with the algorithm of [KS]. Nevertheless, we will present a brief description of the technique, providing enough details to allow us to state the technical difficulty that arises (and was overlooked in [KS]), and to describe a method for overcoming that difficulty.

\subsection{Outline of the Kedem-Sharir Algorithm}

The technique of [KS] is to construct a combinatorial representation of the boundary of the three-dimensional space $F P$ of free placements of $B$, parametrized by $(x, y, \theta)$, where $(x, y)$ are the coordinates of some fixed reference point on $B$, and $\theta$ is the orientation of $B$. The representation of $F P$ is by means of a so-called edge graph $E G$, whose nodes are edges of $F P$, and whose arcs connect pairs of nodes in $E G$ if, for some fixed $\theta$, the edges of $F P$, corresponding to these nodes, meet the cross section $F P_{\theta}$ of $F P$ at $\theta$ in two respective adjacent vertices of $F P_{\theta}$ (see a more detailed definition of $F P_{\theta}$ in the next paragraph). The edge graph preserves the connectivity of $F P$-each connected component of $E G$ consists of all arcs that bound the same connected component of $F P$. Once the edge graph is constructed, motion planning is reduced to path searching in that graph.

The edge graph $E G$ is built incrementally, by sweeping a planar cross section of the form $\theta=$ const over the space $F P$. We denote by $F P_{\theta}$ the cross section of $F P$ at a fixed orientation $\theta$. Observe that $F P_{\theta}$ represents the space of free placements of $B$ when $B$ is allowed only to translate at the fixed orientation $\theta$. As shown, e.g., in [KLPS], the space $F P_{\theta}$ is a planar polygonal region that has only $O(k n)$ edges. In more detail, let $B_{\theta}$ denote the standard placement of $B$ at which it has orientation $\theta$ and its reference point lies at the origin. Assume, without loss of generality, that the obstacles are a collection of convex polygons with pairwise disjoint interiors, and let us denote them by $A_{1}, \ldots, A_{m}$. Let $A_{i}^{*}(\theta)$ denote the Minkowski sum $A_{i} \oplus\left(-B_{\theta}\right)$, for $i=1, \ldots, m$. Then $F P_{\theta}$ is the complement of the union of the expanded obstacles $A_{i}^{*}(\theta)$. It is shown in [KLPS] that the boundaries of any pair of distinct expanded obstacles cross in at most two points, and that this implies that the union of the expanded obstacles has at most $6 m=O(n)$ concave boundary vertices. Hence $F P_{\theta}$ has at most $O(n)$ convex vertices and at most $O(k n)$ concave vertices (which are necessarily corners of the expanded obstacles $A_{i}^{*}(\theta)$ ). Note that each convex vertex of $F P_{\theta}$ represents a placement of $B$ at orientation $\theta$ at which it makes simultaneously two distinct contacts with the obstacles, while otherwise remaining free of collision with the obstacles; each concave vertex of $F P_{\theta}$ represents a placement of $B$ at which a corner of $B$ makes contact with some obstacle corner, while otherwise remaining free.

The construction of $E G$ begins by constructing a vertex graph $V G_{\theta_{0}}$ at the initial orientation $\theta_{0}$ of $B$. This graph represents the boundary of $F P_{\theta_{0}}$ when $B$ is allowed to translate at the fixed initial orientation $\theta_{0}$-its nodes are the vertices of $F P_{\theta_{0}}$ and its arcs connect pairs of adjacent vertices along the boundary of $F P_{\theta_{0}}$ (see [KS] for a more precise formulation). As $\theta$ varies, the vertices of $F P_{\theta}$, as they trace the corresponding edges of $F P$, are followed. Sweeping through $F P$ in this manner, we need to detect critical orientations, at which edges of $F P$ start or end. Roughly speaking, a critical orientation is an orientation of $B$ at which there exists a placement where $B$ makes three simultaneous contacts with the obstacles, while otherwise remaining free. Once the list of all critical 
orientations $\Xi$ is constructed (and sorted), the sweep over $F P$ can be performed, because the critical events that occur during the sweep are already known and the graphs $V G_{\theta}$ and $E G$ can be updated at each critical orientation. Except for various additional technical details, which we omit here, this is a high-level description of the algorithm of [KS]

\subsection{A Technical Difficulty in the Algorithm}

The main task required by the algorithm is thus the efficient computation of all critical orientations. As shown in [LS], the number of critical orientations is $O\left(k n \lambda_{6}(k n)\right)$, where $\lambda_{s}(m)$ is the maximum length of a Davenport-Schinzel sequence of order $s$ composed of $m$ symbols (it is almost linear, and slightly superlinear in $m$ for any fixed $s$; see [HS] and [ASS]).

Our goal is to compute all these orientations in time $O\left(k n \lambda_{6}(k n) \log k n\right)$. The technique of [LS] and [KS] does not achieve this goal exactly, which is the source of the problem we are about to discuss; instead it constructs a superset of all critical orientations. The size of this superset is also $O\left(k n \lambda_{6}(k n)\right)$. Each of the orientations in the superset has a placement at which $B$ makes simultaneously three obstacle contacts, but for some of these orientations the corresponding triple-contact placement might not be free. We do not know of any efficient way to distinguish between the computed orientations whose associated placements are free (we refer to them as valid critical orientations) and those that do not have this property, which we call spurious, as in [KS]. For simplicity, we refer to all computed orientations as critical. Figure 1 shows placements of $B$ at critical orientations. In (a) the critical orientation is valid and in (b) the critical orientation is spurious.

The reason why the algorithm in [KS] may generate spurious critical orientations lies in its technique (adapted from [LS]) for computing critical orientations. We sketch the technique briefly. Define a contact as a pair $O=(W, S)$, where $W$ is an obstacle side or corner and $S$ is a corner or side of $B$, respectively. Given two contacts $O, O^{\prime}$ and an orientation $\theta$, it is shown in [LS] and [KS] that if there is a free placement of $B$ at orientation $\theta$ where it makes a double contact involving $O$ and $O^{\prime}$, then one of these contacts, say $O^{\prime}$, must bound the other in a certain direction, in the following sense: Let $B^{*}$ be the convex hull of the two features of $B$ involved in the contacts $O, O^{\prime}$. Then we

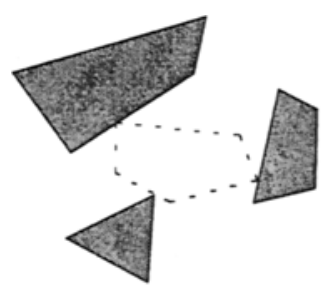

(a)

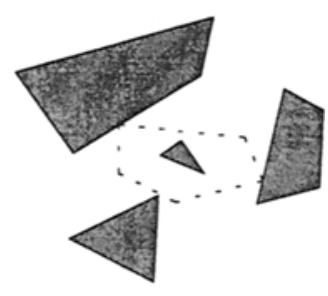

(b)

Fig. 1. (a) A valid critical orientation and (b) a spurious critical orientation; the obstacles are shaded and $B$ is drawn in dashed lines. 


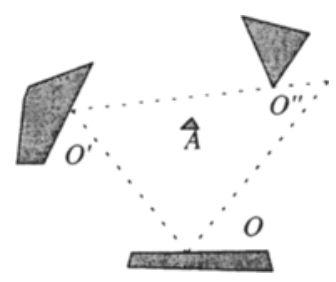

Fig. 2. Obstacle containment not encoded in any bounding function.

can translate an isothetic copy of $B^{*}$ in this direction so that it continues to make the contact $O$ and intersect the obstacle side or corner corresponding to $O^{\prime}$, until it terminates the contact $O$ (at an appropriate comer-corner contact). (See the cited papers for a more detailed definition; see also Fig. 2 where $O^{\prime}$ bounds $O$, and both $O$ and $O^{\prime}$ bound $O^{\prime \prime}$.)

This allows us to introduce a collection of bounding functions $F_{O, O^{\prime}}(\theta)$, each measuring the translation distance along $O$ from one endpoint of $O$ (a corner-corner contact) to the point at which $B$ (at orientation $\theta$ ) makes the double contact $O, O^{\prime}$, where $O^{\prime}$ bounds $O$. These are partial functions of $\theta$, and are defined only when such a double contact is possible and when $O^{\prime}$ bounds $O$ at that contact. It is shown in [LS] and [KS] that by computing various upper and lower envelopes of certain subsets of bounding functions, and by computing various intersections of these envelopes, one can obtain (a superset of) all critical orientations.

The main weakness of this technique is that the bounding functions do not represent all the information about collision of $B$ with the obstacles. A typical problematic example is shown in Fig. 2; in that figure, as $B$ slides along the contact $O$ it always contains the small obstacle $A$, and this fact is not encoded in any bounding function, because there exists no double contact (at this orientation) involving $O$ and a contact of $B$ with $A$. On the other hand, the double contacts $O, O^{\prime}$ and $O, O^{\prime \prime}$ are encoded in their respective bounding functions, hence the critical orientation where $B$ makes the triple contact $O$, $O^{\prime}, O^{\prime \prime}$ is included in the superset of the critical orientations even though it does not represent a free placement of $B$.

As described above, the algorithm of [KS] constructs the edge-graph $E G$ by sweeping over the list of critical orientations (both valid and spurious) and by processing incrementally the changes that occur in $V G_{\theta}$ at each critical orientation $\theta$. The algorithm has to check whether the current critical orientation $\theta$ is valid or spurious, to discard spurious orientations, and to update $V G_{\theta}$ and $E G$ at valid orientations. The problem is to find an efficient technique for checking whether the current orientation is valid or not. (Once the orientation $\theta$ is ascertained to be valid, the updating of $E G$ and of $V G_{\theta}$ is easy and straightforward.)

\subsection{A Partial Solution by Kedem and Sharir}

The paper [KS] suggests the following approach: Suppose a triple contact, involving contacts $O, O^{\prime}, O^{\prime \prime}$, occurs at a critical orientation $\theta$. If, for this orientation, the corresponding placement of $B$ is a limit of free placements of $B$ at orientations $\theta^{\prime}$ sufficiently 
close to, and preceding $\theta$, then $\theta$ is clearly valid. We refer to such $\theta$ 's as having the continuity property. Moreover, the validity of such an orientation is checked in [KS] in logarithmic time as follows: Maintain, for each contact $O$, a dynamic list $L_{o}(\theta)$ of all valid double contacts $O, O^{\prime}$ that occur at the current orientation $\theta$, sorted by their position along $O$ (these are the vertices of $F P_{\theta}$ along the edge defined by $O$ ). If $\theta$ is a critical orientation with the continuity property, whose associated critical placement involves three contacts $O, O^{\prime}, O^{\prime \prime}$, then at least one of the lists $L_{O}(\theta), L_{O^{\prime}}(\theta), L_{O^{\prime \prime}}(\theta)$ must contain at least one of the other two contacts. Thus by simply searching through these lists we can determine whether $\theta$ is valid. (See below and [KS] for some additional details.) However, when the critical orientation $\theta$ is due to the appearance of a new component in $F P_{\theta}$, then the local information needed to determine the validity of $\theta$ is not available in the lists $L_{O}$ of the involved triple contact. Thus the method in [KS] does not apply and we have no way to determine, using only local information, whether $\theta$ is valid or spurious. This is the problem that we consider in this paper.

\subsection{A Costly Solution by Sharir and Toledo}

One recent solution of this problem was proposed by Sharir and Toledo [ST] and [T], who apply the algorithm of [KS] to solve extremal polygon containment problems. They prepare several data structures for triangle range searching and for segment intersection queries, and query these structures with each critical placement of $B$-the placement is free if and only if, at this placement, no corner of $B$ lies inside an obstacle, $B$ does not contain any obstacle corner, and no side of $B$ intersects any obstacle side. All these tests can be done in a total of $O(k \log k n)$ time per placement, allowing sufficient storage for the data structures (which is available anyway in the algorithm of [KS]). This method will cause the algorithm of [KS] to run in time $O\left(k^{2} n \lambda_{6}(k n) \log k n\right)$, which is a factor of $k$ worse than the original time bound in [KS]. If $k$ is a small constant, then this increase in running time is insignificant, but if $k$ is large then this may be costly. Our goal in this note is to find an alternative way of overcoming the problem while retaining the original time bound $O\left(k n \lambda_{6}(k n) \log k n\right)$ of [KS].

\section{Our Solution}

We now describe our solution of this problem. The task at hand is to find all valid critical orientations at which a new component of $F P_{\theta}$ emerges. Once we know them, we will be able to apply the algorithm of [KS], which computes $F P$ and $E G$ incrementally, and to discard in the process spurious critical orientations which are not due to an appearance of a new component of $F P$. Our solution has the feature that, at the end of the construction, we do not necessarily have the description of all the cells (connected components) of $F P$. Specifically, we distinguish between interesting cells and dull cells of FP. Dull cells have the property that all their $\theta$-cross sections are convex and they do not show up at the cross section of $F P$ at the initial orientation $\theta_{0}$ of $B$, whereas interesting cells have at least one nonconvex cross section, or have a nonempty cross section at $\theta_{0}$. This is not a real limitation of the algorithm, because it still guarantees that the cell containing the 
initial placement of $B$ (at orientation $\theta_{0}$ ) is fully constructed. See below for more details concerning this issue.

\subsection{Critical Orientations of Concavity}

In what follows we will refer to the cells of $F P$ by their cross sections with the planes $\theta=$ const at consecutive critical orientations. Let $\theta_{1}$ be an orientation where a new component $C$ of $F P$ emerges (as $\theta$ increases past $\theta_{1}$ ). Assuming that the obstacles and $B$ lie in general position (see [KS] and [LS]), $C$ starts as a point at $F P_{\theta_{1}}$ and grows to a small triangle at cross sections $F P_{\theta}$ for orientations $\theta$ slightly larger than $\theta_{1}$. As $\theta$ increases, more sides can be added to $C$ at further critical orientations. Our main observation is that if, during the process of the incremental construction of $F P, C$ is going to be connected to another connected component of $F_{\theta^{*}}$, at some critical orientation $\theta^{*}$, then at orientations $\theta$ greater than but sufficiently close to $\theta^{*}$, the boundary of $C$ becomes locally nonconvex, i.e., nonconvex in the neighborhood of the triple contact that induces this critical orientation. See, for example, Fig. 3(i), which shows the cross sections before, at, and after an instance of such a merge of two connected components, as $\theta$ sweeps through the corresponding critical orientation $\theta^{*}$.

We will test all the critical orientations at which $C$ becomes locally nonconvex (we refer to them as critical orientations of concavity), to determine whether they are valid or spurious. The determination of all valid critical orientations of concavity will then facilitate a correct computation of all the interesting cells of $F P$.

Let $\theta^{*}$ be the smallest orientation greater than $\theta_{1}$ that is a critical orientation of concavity (for the component $C$ appearing at $\theta_{1}$ ). Of course, $\theta^{*}$ might not exist, but then either $C$ must be dull, or $C$ is nonempty at $F P_{\theta_{0}+2 \pi}$ (where $\theta_{0}$ is the initial orientation of
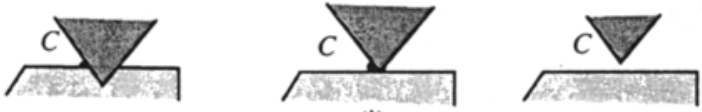

(i)
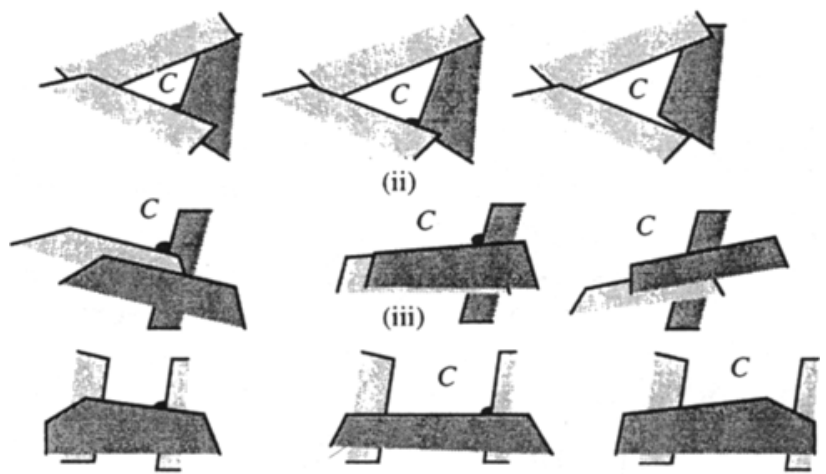

(iv)

Fig. 3. The ways in which $C$ becomes locally nonconvex. The shaded objects are expanded obstacles, and $\theta$ increases from left to right. 
$B$ ), and has only convex cross sections in the interval $\left[\theta^{*}, \theta_{0}+2 \pi\right]$. In the former case we can ignore $C$, since the new algorithm does not have to compute that cell. The latter case is handled by artificially defining the orientation $\theta_{0}+2 \pi$ as a critical orientation of concavity (see Section 2.3).

$C$ can become locally nonconvex in one of the following three cases:

(c1) A vertex of $C$ becomes coincident with a convex corner of an expanded obstacle, which then pulls away from an incident edge of $C$, connects $C$ to another component, and becomes a concave corner of the merged component, see Fig. 3(i).

(c2) A vertex $v$ of $C$ becomes coincident with a convex corner of an expanded obstacle, so that this comer pulls away from one of the edges of $C$ incident to $v$, but the expanded obstacle continues to intersect that edge, thereby exposing the convex corner as a convex corner of $C$, see Fig. 3(ii).

(c3) An edge $e$ of $C$ comes to contain a corner $v$ of an expanded obstacle, so that later either $v$ pulls away from $e$ into $C$, or $e$ bends at $v$ into $C$; in either case, $v$ becomes a concave corner of $C$. See Fig. 3(iii) and (iv). This also includes the case, shown in Fig. 3(iii), of two parallel edges of two respective expanded obstacles that become coincident and then "swap" their relative positions. We refer to this vertex of $F P$ as an endpoint of a "crease" that newly appears on the boundary of $F P$.

We claim that these are the only possible ways in which $C$ can become nonconvex. Indeed, for $C$ to be nonconvex, it must contain a comer $v$ of some expanded obstacle $A^{*}(\theta)$ on its boundary, and this corner must reach the boundary of $C$ from outside $C$. If, at the time of transition, $v$ coincides with a vertex of $C$ (that also existed before the critical orientation), then, by the general position assumption, one edge of $A^{*}(\theta)$ incident to $v$ must overlap an edge of $C$. It is then easy to check that, depending on the orientation of the other edge of $A^{*}(\theta)$ incident to $v$, we are either in case (c1) or in case (c2).

The remaining possibility is that $v$ lies on (the relative interior of) an edge $e$ of $C$. We claim that it is impossible that both sides of $A^{*}(\theta)$ incident to $v$ lie outside $C$ (as depicted in Fig. 4(i)). Indeed, suppose to the contrary that this does happen; let $A^{*}$ be the expanded obstacle having $v$ as a corner, and let $\tilde{A}^{*}$ be the expanded obstacle having $e$ as (a portion of) a side. Note that at $\theta^{*}$, and thus also at orientations $\theta$ slightly smaller than $\theta^{*}$, at least some portion of $A^{*}(\theta)$ lies inside $\tilde{A}^{*}(\theta)$. Moreover, $A^{*}\left(\theta^{*}\right)$ must be fully contained in $\tilde{A}^{*}\left(\theta^{*}\right)$, for otherwise their boundaries would have to intersect at least twice, which then implies that at $\theta$ slightly larger than $\theta^{*}$ the boundaries of $A^{*}(\theta)$ and of $\tilde{A}^{*}(\theta)$ would have to intersect in at least four points (the two former points and two new points near $v$ ), contradicting the properties of expanded obstacles, as outlined in Section 1.1 (see also [KLPS]). However, since $A$ and $\tilde{A}$ have pairwise disjoint (and nonempty) interiors, it is impossible for $A^{*}\left(\theta^{*}\right)$ to be fully contained in $\tilde{A}^{*}\left(\theta^{*}\right)$ : Let $\ell$ be a line separating $A$ and $\tilde{A}$; suppose, without loss of generality, that $\ell$ is the $y$-axis and that $A$ lies on its left side. Then, using properties of Minkowski sums, it is easy to show that the leftmost corner of $A^{*}(\theta)$ cannot lie inside $\tilde{A}^{*}\left(\theta^{*}\right)$. This contradiction shows that we thus only have to consider cases where at least one side of $A^{*}\left(\theta^{*}\right)$ incident to $v$ overlaps the edge $e$ of $C$ containing $v$.

If both edges of $A^{*}\left(\theta^{*}\right)$ overlap $e$, then $v$ is not yet a corner of $A^{*}\left(\theta^{*}\right)$, but is about to "bend out" and become such a corner when $\theta$ slightly increases. It is easily seen that 


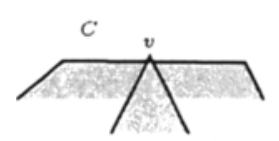

(i) Impossible $A^{*}(\theta)$

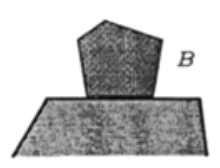

(ii) Possible $A$

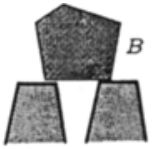

(iii) Degenerate

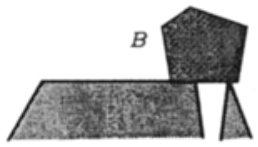

(iv) Degenerate

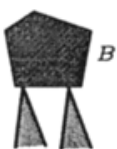

(v) Possible

Fig. 4. Possible and impossible contacts where $v$ lies on the relative interior of an edge of $C$. Case (i) is drawn in configuration space, whereas cases (ii)-(v) are drawn in the original plane.

this happens only when $\theta^{*}$ is an orientation at which a side of $B$ becomes parallel to an obstacle side (and this side of $B$ can touch this obstacle side so that $B$ and the obstacle have disjoint interiors). This is the situation depicted in Fig. 3(iv), and is also illustrated in Fig. 4(ii). Note that there are only $O(k n)$ critical orientations of this kind.

If only one edge of $A^{*}\left(\theta^{*}\right)$ incident to $v$ overlaps $e$, then we have a situation where two edges $e_{1}, e_{2}$ of two respective and distinct expanded obstacles $A_{1}^{*}\left(\theta^{*}\right), A_{2}^{*}\left(\theta^{*}\right)$ overlap (so that both obstacles lie in the same half-plane bounded by the line containing $e_{1}$ and $e_{2}$ ). By the general position assumption, it is impossible that both $e_{1}$ and $e_{2}$ are Minkowski sums involving two (distinct) obstacle edges, because then these edges would have to be parallel (and even collinear), which is ruled out in general position (see Fig. 4(iii)). Similarly, it is impossible that $e_{1}$, say, involves an obstacle edge $W$ and $e_{2}$ a side $S$ of $B$, because, at this orientation, the only possible contact of $B$ with $W$ is then the edge $S$ overlaps $W$ and passes through a vertex of another obstacle, again a situation that does not arise in general position (see Fig. 4(iv)).

We are thus left with the case that $e_{1}$ and $e_{2}$ are Minkowski sums involving the same side $S$ of $B$ and two respective corners $W_{1}, W_{2}$ of two distinct obstacles $A_{1}, A_{2}$. This case can arise (even in general position), as is illustrated in Fig. 4(v), and is the one giving rise to the transition depicted in Fig. 3(iii). The critical orientation $\theta^{*}$ is the orientation of $B$ at which $S$ is parallel to the line $W_{1} W_{2}$. Thus there are only $O\left(k n^{2}\right)$ such orientations.

\subsection{Computing the Critical Orientations of Concavity}

In all the possible ways for $\theta^{*}$ to be a critical orientation of concavity, $B$ must make at $\theta^{*}$ two obstacle contacts, one of which involves contact of a corner of $B$ at an obstacle corner (which induces the expanded obstacle corner "piercing" into $C$ ). There are $O(k n)$ such corner-corner contacts, and we can process each of them, in time $O(k n \log k n)$, to find all the corresponding free critical placements of $B$. To do so, we note that such a corner-corner contact leaves only one degree of freedom for $B$, namely, rotation about the point of contact. For each corner or side $S$ of $B$ and for each obstacle $A_{i}$ we compute the set of orientations at which $S$ meets $A_{i}$ (while $B$ rotates about the fixed corner-corner contact). The sets of orientations where $S$ intersects obstacles are called forbidden sets. Similarly, for each corner or side $W$ of any obstacle we compute the set of orientations at which $B$ meets $W$ (while maintaining the corner-corner contact). It is easily checked that these sets consist of a total of $O(k n)$ angular intervals, and that they can all be computed in time $O(k n)$. We now compute the union of the forbidden sets, using a 
simple sorting process that takes $O(k n \log k n)$ time, and the endpoints of the angular intervals forming the union are precisely the (valid!) critical orientations of concavity that we seek. Repeating this step for each corner-corner contact, the total running time of this step is $O\left((k n)^{2} \log k n\right)$, and it produces the subset $\Xi^{\prime} \subseteq \Xi$ of all the valid critical orientations of concavity.

\subsection{Tracing Events of Concavity Backward in $\theta$ Along FP}

In this subsection we show how we trace critical orientations of concavity backward in $\theta$, to detect the set $\Xi^{\prime \prime}$ of all the valid critical orientations at which new components of intersecting cells emerge (in the forward $\theta$-direction).

Let $\theta^{*} \in \Xi^{\prime}$ be a critical orientation of concavity. Let $v$ be the specific vertex of $C$ at $F P_{\theta^{*}}$ which is involved in this critically, such that $v$ also continues to appear as a vertex of $F P_{\theta}$, for orientations $\theta$ slightly smaller than $\theta^{*}$. The vertex $v$ is defined as follows: In cases (c1) and (c2) we take $v$ to be a vertex of $C$ that is about to become coincident with the convex corner of the corresponding expanded obstacle. In case (c3) we take $v$ to be an endpoint of the edge of $C$ through which the new expanded obstacle corner is about to pierce. See Fig. 3 where these vertices are highlighted. In cases (c1) and (c2) $v$ is readily determined from the local information about the critical configuration arising at $\theta^{*}$, but in case (c3) more work has to be done to compute $v$ (because $v$ is not involved in the critical contact determining $\theta^{*}$ ). We will describe this step in detail below, but for now let us assume that the vertex $v$ is available.

Imagine now that we reverse the direction of the sweep in $\theta$, over the list of critical orientations, starting at $\theta^{*}$ and proceeding in the list backward until $C$ shrinks to a point and disappears. As this sweep begins, $v$ traces an edge of $F P$, which may end at a vertex $w$ of $F P$ (at some other critical orientation). Three situations can then arise:

(a) $w$ is adjacent to at least one (and at most two) new edges of $F P$ emerging from it in the direction of decreasing $\theta$. In this case we continue the tracing along (any of) these edges.

(b) $w$ is a local $\theta$-minimum of $F P$; in other words, the component $C$ that we trace shrinks to $w$ and then vanishes. In this case we add $w$ to the output list $\Xi^{\prime \prime}$.

(c) $w$ is not a local $\theta$-minimum of $F P$, but no edge of $F P$ emerges from $w$ in the direction of decreasing $\theta$. Informally, $w$ is an endpoint of a "crease" along the boundary of $F P$. As argued in Section 2.1, in such a case $w$ must be incident to two collinear (possibly overlapping) edges of expanded obstacles (see case (c3) above). In other words, $w$ is a vertex of $F P$ at which some $\theta$-cross section of $F P$ becomes locally nonconvex (as we sweep forward in $\theta$ ). In this case we can stop the current tracing from $w$, because our procedure will initiate another backward tracing from $w$, in the same manner as in the current description.

The backward sweep in $\theta$ can be implemented in the following manner, similar to that used in [KS]. For each contact $O$ we maintain a sorted list $L_{O}=L_{O}(\theta)$ of contacts, so that for each $O^{\prime} \in L_{O}(\theta)$ the two contacts $O$ and $O^{\prime}$ induce a vertex of $F P_{\theta}$ (or, equivalently, induce an edge of $F P$ that crosses the cross section $F P_{\theta}$ ). Not all such vertices will be maintained, but those maintained are guaranteed to be valid. The lists 
$L_{O}(\theta)$ are maintained as balanced search trees so that searches and updates in any of them can be performed in $O(\log k n)$ time. We initialize these lists by computing the whole cross section $F P_{\theta_{0}}$ at the initial orientation $\theta_{0}$, as in [KS]. We then sweep from $\theta_{0}$ backward through the sorted list $\Xi$ of all critical orientations.

If the current orientation $\theta^{*}$ is a valid critical orientation of concavity (i.e., $\theta^{*} \in \Xi^{\prime}$ ), we find the corresponding vertex $v$ of the corresponding component $C$ of $F P_{\theta^{*}}$ (in a manner to be described shortly), and the two contacts $O, O^{\prime}$ that induce $v$. We add $O^{\prime}$ to $L_{O}$ and $O$ to $L_{O^{\prime}}$. (In case (c1) we will add both vertices that "emerge" from the critical placement, because $C$ might lie on either side of this placement; see Fig. 3(i).)

Suppose that $\theta^{*}$ is some other critical orientation (i.e., $\theta^{*} \in \Xi-\Xi^{\prime}$ ), and that it corresponds to a simultaneous triple contact involving the contacts $O, O^{\prime}, O^{\prime \prime}$. We search with $O^{\prime}$ and $O^{\prime \prime}$ in $L_{O}$, with $O$ and $O^{\prime \prime}$ in $L_{O^{\prime}}$, and with $O$ and $O^{\prime}$ in $L_{O^{\prime \prime}}$. If none of these searches is successful, we ignore $\theta^{*}$, as it does not affect the structure of the lists that we are maintaining. Suppose then that some of these searches succeed, say we find $O^{\prime}$ in $L_{O}$ (and, symmetrically, also $O$ in $L_{O^{\prime}}$ ). The important observation is that $\theta^{*}$ must then be a valid critical orientation, because the critical placement it represents is a limit placement of free placements of $B$ at orientations $\theta \downarrow \theta^{*}$. We thus determine the local changes in $F P_{\theta}$ that occur around the corresponding critical placement, and update the relevant lists $L_{O}, L_{O^{\prime}}, L_{O^{\prime \prime}}$ as needed. These updates may consist of insertions, deletions, or replacements; they can be directly inferred from the local geometric information describing the critical placement. (Since we are assuming here that $\theta^{*}$ is not a critical orientation of concavity, local updating about the critical placement suffices.) These updates ensure that the lists $L$ continue to contain only valid entries after any modification. Each such modification is performed in time $O(\log k n)$.

If the local change at $\theta^{*}$ involves a small triangular component of $F P_{\theta}$ that shrinks at $\theta^{*}$ to a point and then vanishes, then, as already mentioned above, we add $\theta^{*}$ to the output list $\Xi^{\prime \prime}$. We proceed with this sweep until all critical orientations are exhausted. The final output list $\Xi^{\prime \prime}$ gives us all valid critical orientations at which (in the forward sweep in $\theta$ ) interesting new components of $F P_{\theta}$ emerge. The correctness of this procedure follows from the invariant, easy to establish by induction, that (a) the procedure maintains only valid contacts in the lists $L_{O}$; and (b) the procedure traces every path $\pi$ that consists of a sequence of edges of $F P$, is monotonically decreasing in $\theta$, and starts at any designated vertex $v$ of some critical orientation of concavity, or at the orientation $\theta_{0}$. This invariant is easily seen to imply the asserted correctness.

\subsection{Jumping from Endpoints of Creases}

It remains to describe an efficient technique for jumping from an endpoint $v$ of a crease to a vertex along the appropriate edge of the current cross section of $F P$. Recall that this has to be done at critical orientations $\theta^{*}$ of concavity where the corresponding critical placement is of type (c3). By the analysis given in Section 2.2, there are only two subcases to consider:

(a) $v$ is about to become a corner of some expanded obstacle $A^{*}$; the two edges of $A^{*}\left(\theta^{*}\right)$ are currently collinear and are about to bend and to expose $v$ as a vertex. 


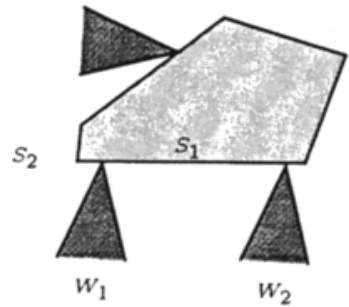

(i)

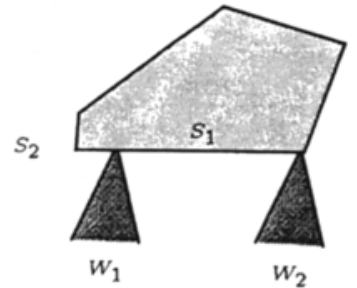

(ii)

Fig. 5. Jumping from an endpoint of a crease (in physical space); (i) the jump terminates at a third obstacle contact; (ii) the jump terminates at another critical placement of concavity. In both cases $B$ is translated to the left, from the critical placement of concavity where the corner $S_{2}$ touches $W_{1}$.

As noted above, there are only $O(k n)$ such critical placements, so we can afford to spend $O(\mathrm{kn})$ time in processing each of them. This allows us to compute the desired endpoints of the edge of $F P_{\theta^{*}}$ containing $v$ using a straightforward brute-force technique.

(b) $v$ represents a free placement at which a side $S_{1}$ of $B$ touches two wall corners $W_{1}$ and $W_{2}$, and the contact with one of the wall corners, say $W_{1}$, occurs at an endpoint of $S_{1}$. In physical space, the task at hand is to translate $B$ so that $S_{1}$ maintains contact with both $W_{1}$ and $W_{2}$, and to find the first placement at which this translation stops being free. This happens either when a third contact of $B$ with an obstacle occurs, as in Fig. 5(i), or when the other endpoint of $S_{1}$ touches one of the wall corners, as in Fig. 5(ii). Clearly, this translation of $B$ can be made in only one direction; translating in the opposite direction would immediately lose one of the contacts of $S_{1}$ with $W_{1}, W_{2}$.

We make the observation that if $\theta^{*}$ is a valid orientation, then, in either of the subcases of (b), the terminal placement is a free triple-contact placement, and thus is encoded in the list $\Xi$ of all critical placements. By the general position assumption, the only two critical placements with the same critical orientation $\theta^{*}$, are the original critical orientation of concavity and the one encountered while translating $B$ as above, and so that other placement is readily available from the (sorted) list $\Xi$ of all critical orientations. In other words, we can execute this jump in $O(\log k n)$ time.

To recap, the overall cost of executing the jumps from endpoints of creases of $F P$ is $O\left(k^{2} n^{2}+k n^{2} \log k n\right)$, which is subsumed by the cost of the other steps of the algorithm.

\subsection{Computing the Interesting Cells of FP}

After identifying in this manner all valid critical placements where new interesting components truly emerge, we now apply the original algorithm of [KS], with the following modification. When we reach a critical orientation $\theta^{*}$, we check if it is an orientation where a new component of $F P_{\theta}$ (potentially) emerges. If so, we check if $\theta^{*}$ belongs to $\Xi^{\prime \prime}$. If not, we ignore $\theta^{*}$ because it must be spurious or involve a dull component. If $\theta^{*}$ does belong to $\Xi^{\prime \prime}$, we insert the new resulting vertices of $F P_{\theta}$ into the appropriate lists 
$L_{O}$ of contacts. If $\theta^{*}$ does not involve a newly emerging component, and is defined by three contacts $O, O^{\prime}, O^{\prime \prime}$, we search with each of these contacts in the lists of the two other contacts, as in the preceding backward-sweeping stage. If none of these searches succeed, it might still be the case that $\theta^{*}$ is the starting endpoint of a crease in $F P$ and is thus a valid critical orientation of concavity, and is stored in the list $\Xi^{\prime}$. If so, we update $F P_{\theta}$ as above. Otherwise, we discard $\theta^{*}$, since it is spurious or corresponds to a placement in a dull component. If some of the searches succeed, we know that $\theta^{*}$ is valid, and update the lists as required, using the local data concerning the critical placement that $\theta^{*}$ represents.

We claim that this modified algorithm correctly traces all the edges of interesting components of $F P$. Indeed, the algorithm maintains the invariant that, at any orientation $\theta$, its data structures represent all true vertices of $F P_{\theta}$ that lie in interesting components, and only those vertices. We omit a full proof of this fact, which is a consequence of the analysis given in $[\mathrm{KS}]$ and of the discussion above.

There is also the issue of constructing the edge graph $E G$ so that it captures the connectivity of (the interesting portion of FP, as outlined in Section 1.1. This can be done in much the same way as in [KS], and we refer the reader to that paper for details.

To summarize, we propose to use the following four-stage algorithm:

- Compute (a superset of) all critical orientations, as in [LS] and [KS]. This takes time $O\left(k n \lambda_{6}(k n) \log k n\right)$.

- Compute all critical orientations of concavity, as described above. This takes time $O\left((k n)^{2} \log k n\right)$.

- Perform the backward sweep in $\theta$, as described above, to obtain the list of all valid critical orientations where new components of $F P_{\theta}$ emerge (in the forward $\theta$-direction).

- Finally, apply the algorithm of [KS], modified as above. This step, as well as the preceding one, takes time $O\left(k n \lambda_{6}(k n) \log k n\right)$.

The actual motion planning step is part of the final step, and is performed just as described in [KS]. Hence, we have shown that the modified algorithm performs the desired motion planning in time $O\left(k n \lambda_{6}(k n) \log k n\right)$, and is thus as efficient asymptotically as the algorithm of $[\mathrm{KS}]$.

Remark. The data structures produced by the above algorithm are also useful if we change the initial (and final) placements of $B$. Given a new initial placement $Z$ of $B$, we try to locate $Z$ in the interesting portion of $F P$ (by brute force). If $Z$ lies in an interesting cell, motion planning can then proceed using the final graph searching step of [KS]. Otherwise, $Z$ must lie in a dull cell. In this case, we translate $Z$ to a placement $Z^{\prime}$ lying on an edge of $F P$, and start tracing edges of $F P$ from $Z^{\prime}$ in both increasing and decreasing $\theta$-directions. This is done as in the step, described above, of tracing from critical orientations of concavity, and is only simpler, because no critical orientations of concavity will be encountered during this tracing, as $Z$ lies in a dull cell. We stop the tracing when we reach any placement whose orientation is equal to that of the final placement, and then test whether these two placements can be connected by a free straight-line translational motion. If so, we have found a free motion connecting between the new initial and final placements. If not, no such motion is possible. While the 
running time of this step may be asymptotically the same as that of the whole algorithm, in practice this step can be expected to run much faster.

\section{Acknowledgments}

We wish to thank Joe O'Rourke and two anonymous referees for detailed and constructive comments on an earlier version of this paper. Their comments led to significant improvements in the results and presentation of the paper.

\section{References}

[ASS] P. Agarwal, M. Sharir, and P. Shor, Improved bounds for the length of general Davenport-Schinzel sequences, J. Combin. Theory Ser. A 52 (1989), 228-274.

[HS] S. Hart and M. Sharir, Nonlinearity of Davenport-Schinzel sequences and of generalized path compression schemes, Combinatorica 6 (1986), 151-177.

[KLPS] K. Kedem, R. Livne, J. Pach, and M. Sharir, On the union of Jordan regions and collision-free translational motion amidst polygonal obstacles, Discrete Comput. Geom. 1 (1986), 59-71.

[KS] K. Kedem and M. Sharir, An efficient motion-planning algorithm for a convex polygonal object in two-dimensional polygonal space, Discrete Comput. Geom. 5 (1990), 43-75.

[LS] D. Leven and $M$. Sharir, On the number of critical free contacts of a convex polygonal object moving in 2-D polygonal space, Discrete Comput. Geom. 2 (1987), 255-270.

[ST] M. Sharir and S. Toledo, Extremal polygon containment problems, Comput. Geom. Theory Appl. 4 (1994), 99-118.

[T] S. Toledo, Extremal polygon containment problems, Proc. 7th Ann. Symp. on Computational Geometry, 1991, pp. 176-185.

Received January 23, 1995, and in revised form June 13, 1996. 